\title{
All change: protein conformation and the ubiquitination reaction cascade
}

\author{
Christiane Riedinger and Jane A Endicott*
}

\author{
Address: Laboratory of Molecular Biophysics and Department of Biochemistry, South Parks Road, Oxford OX1 3QU, UK \\ *Corresponding author: Jane A Endicott (jane.endicott@bioch.ox.ac.uk) \\ FI000 Biology Reports 2009, I:19 (doi: 10.3410/BI-19) \\ The electronic version of this article is the complete one and can be found at: http://FI000.com/Reports/Biology/content/I/I9
}

\begin{abstract}
The structures of enzymes that collectively modify proteins by covalent addition of ubiquitin-like protein moieties have provided significant insights into the regulatory pathways they compose and have highlighted the importance of protein flexibility for the mechanism and regulation of the ubiquitination reaction.
\end{abstract}

\section{Introduction and context}

Protein modification by the addition of a small ubiquitin-like protein (Ubl) is a mechanism widely employed in eukaryotic cells to control protein activity after translation (reviewed in [1]). Ubls constitute a growing family of structurally similar molecules that include ubiquitin (Ub), NEDD8 (neural precursor cell expressed, developmentally downregulated 8), SUMO (small ubiquitin-like modifier), ISG15 (interferon-stimulated gene 15), and ATG8 (autophagy 8), and are reviewed in [2]. Ubls become conjugated to target proteins in a three-enzyme cascade. In the first reaction, an E1 (Ubl-activating) enzyme transfers an adenyl moiety from ATP to the C-terminal glycine residue of the Ubl, thus activating the Ubl for subsequent transfer to an E1 cysteine residue to generate a thioester linked E1-Ubl conjugate. In the next reaction, the Ubl is transferred to an E2 (Ubl-conjugating enzyme) active site cysteine residue, while in the final reaction, an E3 (Ubl ligase) catalyses transfer of the activated Ubl to its protein target (reviewed in [3-5]). In some cases, targets are modified by the addition of only a single Ubl, but in others, signal diversity is increased by the addition of chains of Ubls in which Ubl identity, chain length, and linkage type all contribute to specificity (reviewed in $[6,7])$.
Like many proteins involved in signalling pathways, E1, E2 and E3 enzymes are characterised by modular architectures in which regulator binding and enzymatic activities are segregated either to different domains within the same protein or, as is the case with many E3s, to different proteins. Recent structures determined by X-ray crystallography have provided detailed snapshots of E1-, E2-, and E3-containing complexes caught at different stages in their catalytic cycles and from these structures important insights into their mechanisms have been gained.

\section{Major recent advances}

The recent determination of the structure of a complex containing the NEDD8-E1 enzyme APPBP1-UBA3, a catalytically inactive variant of its cognate E2 (Ubc12), two NEDD8 molecules and Mg-ATP [8] (Figure 1a) elegantly demonstrated how a conformational change in an E1 can generate an affinity switch; only when the E1 is doubly loaded with its cognate Ubl does it acquire high affinity for its cognate E2. In this example, the APPBP1UBA3 E1, once charged with two NEDD8 molecules at its adenylation (A)- and thioester (T)-active sites, transfers the (covalently bound) T-site NEDD8 to Ubc12 (an E2) and then adopts a conformation that promotes the release of the E2-Ubl conjugate. As the E2-binding sites for E1 and for E3 are mutually exclusive, the structure suggests that the E2 must dissociate from the E1 before it 
Figure I. Conformational change accompanies catalysis in EIs

(a)

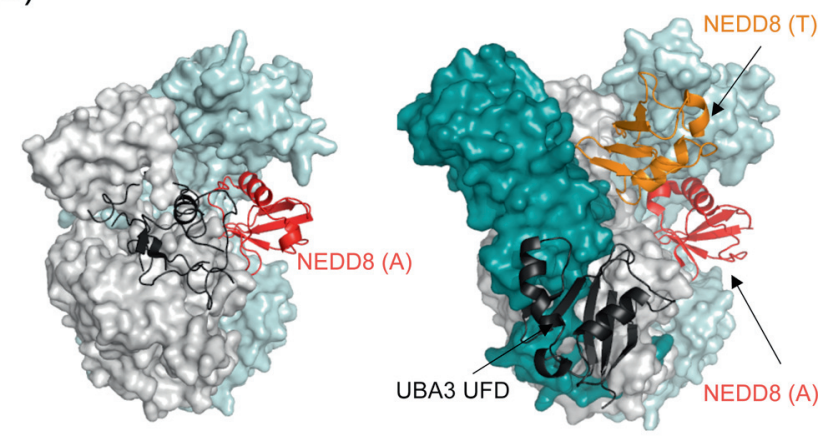

(b)

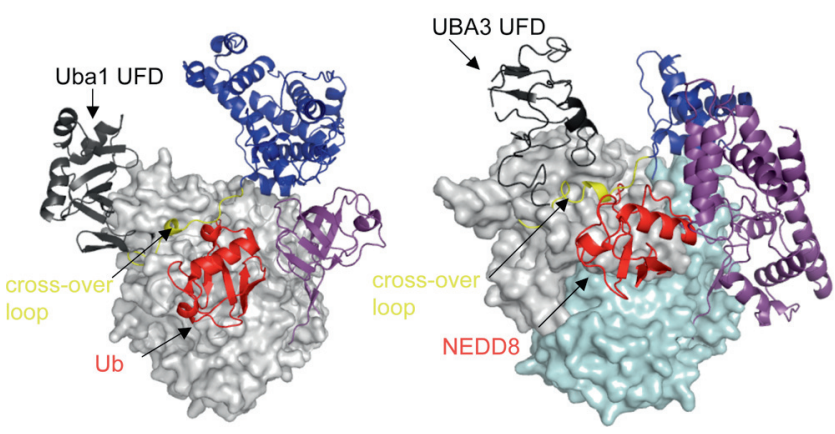

(a) A conformational switch accompanies double-loading of an EI. A comparison of the structure of a singly loaded EI complex (APPBPI-UBA3NEDD8-ATP, PDB code IR4N [I I], left-hand side) and that of the doubly loaded complex (PDB code 2NVU [8], right-hand side) elegantly demonstrates the conformational switch within the EI that accompanies double-loading of the EI with its cognate Ubl. The direction of the rearrangement of the UFD domain of UBA3 (that binds to the E2) as well as that in which the NEDD8 is passed on from the A site to the T site are indicated with arrows. The molecular surfaces of APPBPI and UBA3 are highlighted in light cyan and grey, respectively. In the right-hand panel, the E2 $\mathrm{Ubcl} 2$ is represented in turquoise. The UBA3 UFD domain (black) is shown in ribbon representation. Bound NEDD8 molecules are coloured red (A site) and orange ( $\mathrm{T}$ site). (b) Comparison of singly charged EI structures. Left panel: Ubal-Ub complex (PDB code 3CMM [10]). Right panel: APPBPI-UBA3-NEDD8-ATP complex. The bound small ubiquitinlike protein (Ub or NEDD8, respectively) is coloured red and the UFD domains of both $\mathrm{EI}$ enzymes are coloured black. Within the EI structures, the two juxtaposed catalytic cysteine half domains that compose the catalytic cysteine domain [19] are coloured pale yellow and light blue (respectively), the crossover loop that connects the adenylation and catalytic cysteine domains is coloured dark blue, and the molecular surface of the rest of the El is coloured grey (Ubal and UBA3) and light cyan (APPBPI). A, adenylation; APPBPI, amyloid beta precursor protein-binding protein I; NEDD8, neural precursor cell expressed, developmentally downregulated 8; PDB, Protein Data Bank; T, thioester; Ub, ubiquitin; Ubl, small ubiquitin-like protein; UBA3, ubiquitin-activating enzyme 3; UFD, ubiquitin-fold domain. can associate with a cognate E3. Indeed, enzymological studies, at least in some cases, have shown that this is in fact the case [9].

Subsequent determination of the structure of Uba1, a yeast Ub-E1 non-covalently bound to $\mathrm{Ub}$, has demonstrated that the E1 mechanism, though broadly conserved, is subject to variation in detail [10] (Figure 1b). A comparison of the structures of the two copies of the Uba1-Ub complex in the crystal asymmetric unit further highlights the different positions that the ubiquitin-fold domain (UFD) of the E1 can adopt relative to the other E1 domains. Notably, in this singly Ub-loaded structure, the location of the UFD is similar to that of the UFD in the doubly NEDD8-loaded APPBP1-UBA3 structure and distinct from that observed in structures of NEDD8 [11] and SUMO [12] E1 enzymes singly Ubl-loaded at the A site. As discussed in Huang et al. [8], the reorientation of the UFD that accompanies double-loading of the NEDD8 E1 with NEDD8 is critical to subsequent transfer of the covalently bound T-site NEDD8 to the E2. In addition, the canyon formed between the UFD and the catalytic cysteine domain in the Uba1-Ub structure (Figure 1b) is much wider than that seen in APPBP1UBA3. Taken together, these and other observations suggest that the conformational changes that accompany transfer of Ub from E1 to E2 will be much less dramatic than those associated with transfer of NEDD8 or SUMO.

As with E1 and E2 enzymes, an overriding theme to emerge from recent structural studies of E3s is the functional importance of conformational flexibility. E3s fall into three major subclasses according to the identity of the domain that binds the covalent E2-Ubl complex. These subclasses are referred to as 'homologous to the E6-AP carboxyl terminus' (HECT), 'really interesting new

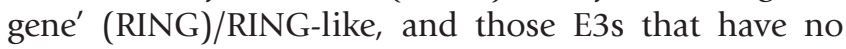
resemblance to the HECT- or RING-containing enzymes. Recent structural and biochemical studies have begun to elucidate the mechanistic diversity of this broad enzyme class (reviewed in [4]), but in all examples, a recurrent theme is the importance of conformational flexibility to allow the E3 complexes to bind and modify different protein substrates.

A particular example, well documented by structural studies, is that of the SCF [Skp1 (S-phase kinaseassociated protein 1)-cullin-F box] E3 Ub ligase family (reviewed in [13]). This E3 contains a RING domain that binds to the cullin subunit. The cullin functions as a scaffold within the complex and also binds to the adaptor protein Skp1 [14]. SCF activity is promoted by cullin neddylation [15] and inhibited by CAND1 (cullinassociated and neddylation-dissociated 1) binding [16]. 
Skp1 and the cullin together mediate the interaction between a diverse set of F-box-containing proteins that bring substrates to the E2, and E3 catalytic core. Models for SCF E3/E2 complexes have been constructed by superimposing structures of selected subcomplexes $[14,17]$ (Figure 2a). However, it is difficult to see how catalysis will proceed as the E3 substrate bound to its cognate F-box protein is located approximately $50 \AA$ away from the bound activated E2-Ubl [14].

The structure of a neddylated cullin-Rbx1 (ring-box 1) complex [17] (Figure 2b) and enzymological studies of selected SCFs $[15,18]$ together provide valuable extra information to help solve this puzzle. Previous attempts to generate models for an SCF E3 complex had employed structures that contained a non-neddylated cullin subunit. A comparison of the neddylated and non-neddylated cullin structures bound to Rbx1 reveals that neddylation leads to the adoption by the cullin-Rbx 1 complex of a more open structure in which the Rbx1 $\mathrm{N}$-terminal linker is no longer constrained by interactions with the cullin, and as a result, the Rbx1 RING domain is also freed so that bound substrate is predicted to come significantly closer to charged E2 [17]. This prediction is supported by the fact that the neddylated $\mathrm{SCF}^{\beta-T r C P}$ complex, but not the non-neddylated form of this complex, promotes the BMPS [N-(beta-maleimidopropyloxy)succinimide ester]-mediated crosslinking of $\mathrm{UbcH} 5 \mathrm{c}$ (an SCF ${ }^{\beta-\operatorname{TrCP}} \mathrm{E} 2$ ) with a peptide from $\beta$-catenin (an $\mathrm{SCF}^{\beta-\operatorname{TrCP}}$ substrate) [15].

As a further result of these structural changes, the cullin CAND1-binding site is also lost. Thus, the structure of the neddylated cullin-Rbx1 complex answers the question of how the distance between the bound substrate and E2 might be spanned and of how CAND1 inhibitor binding and cullin neddylation reciprocally regulate SCF activity: CAND1 binding stabilises the closed (inactive) cullin structure, whereas cullin neddylation promotes the more open (active) conformation and simultaneously prevents CAND1 association.

\section{Future directions}

Now that initial structures have given a glimpse of the structural diversity of the enzymes of the Ubl conjugation pathway, future work will focus on characterising complete complexes at different stages of their catalytic cycles. We expect further insights into this important signalling mechanism to emerge from visualising and characterising both the conserved and the unique mechanistic aspects of different Ubl-conjugating enzymes.
Figure 2. Conformational change accompanies catalysis in E3s
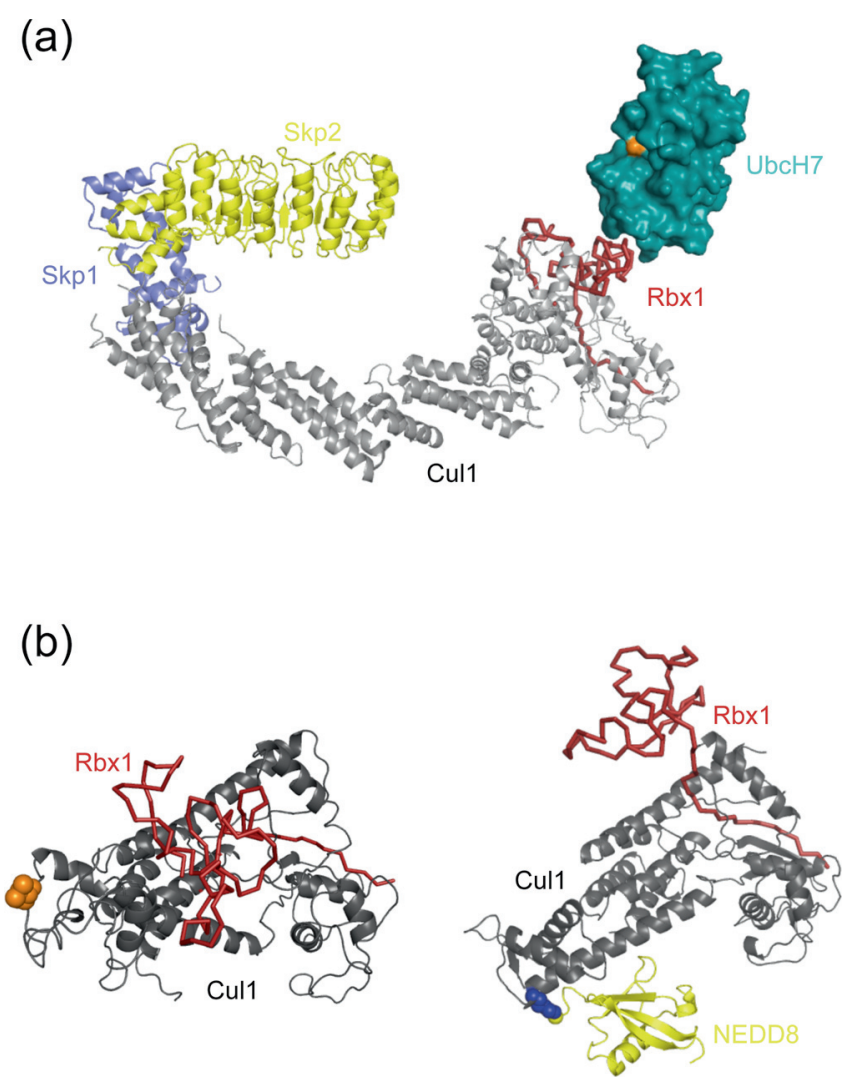

(a) Model for an E2/SCF $F^{\text {Skp2 }}$ complex. This image was generated by merging the structures of Cull-RbxI-Skp I-Skp2 (ILDK) [I4] and Skp I/Skp2 (IFQV) [20] as described in [14]. The E2 UbcH7 (2FBV) was then placed on to the RbxI RING domain guided by the c-Cbl-UbcH7 structure [2I]. Skpl and Skp2 are shown in blue and yellow (respectively), Cull in dark grey, Rbxl in dark red, and the E2 $\mathrm{UbcH} 7$ in cyan. The catalytic cysteine is coloured pink. (b) Conformational change accompanies neddylation of Cull. The panels highlight the change in the association of Rbxl with Cull that accompanies Cull neddylation (dark red arrow). For comparison, only the Cull C-terminal domain (dark grey) is drawn. The left panel shows the non-neddylated structure in which Rbxl (dark red ribbon) forms a close association with Cull. Lys724 (non-neddylated) is drawn as blue spheres. The right panel depicts the neddylated Cull-Rbxl complex. Neddylation (NEDD8, red) promotes a more open Cull-RbxI association. Lys724 covalently bound to NEDD8 is drawn as blue spheres. Cull, cullin I; NEDD8, neural precursor cell expressed, developmentally downregulated 8; RbxI, RIGG-box protein I; RING, really interesting new gene; SCF, Skp I [S-phase kinase-associated protein I]-cullin-F box; Skp, S-phase kinaseassociated protein.

\section{Abbreviations}

A, adenylation; CAND1, cullin-associated and neddylation-dissociated 1; HECT, homologous to the E6-AP carboxyl terminus; NEDD8, neural precursor cell 
expressed, developmentally downregulated 8; Rbx1, ring-box protein 1; RING, really interesting new gene; SCF, Skp1 [S-phase kinase-associated protein 1]-cullin-F box; Skp1, S-phase kinase-associated protein 1; SUMO, small ubiquitin-like modifier; $\mathrm{T}$, thioester; Ub, ubiquitin; UBA3, ubiquitin-activating enzyme 3; Ubl, small ubiquitin-like protein; UFD, ubiquitin-fold domain.

\section{Competing interests}

The authors declare that they have no competing interests.

\section{Acknowledgements}

The authors thank $M$ Noble for his critical reading of the manuscript and the Medical Research Council (UK) for financial support.

\section{References}

I. Hershko A, Ciechanover A: The ubiquitin system. Annu Rev Biochem 1998, 67:425-79.

2. Kerscher $O$, Felberbaum $R$, Hochstrasser M: Modification of proteins by ubiquitin and ubiquitin-like proteins. Annu Rev Cell Dev Biol 2006, 22: 159-80.

3. Capili AD, Lima CD: Taking it step by step: mechanistic insights from structural studies of ubiquitin/ubiquitin-like protein modification pathways. Curr Opin Struct Biol 2007, 17:726-35.

4. Dye BT, Schulman BA: Structural mechanisms underlying posttranslational modification by ubiquitin-like proteins. Annu Rev Biophys Biomol Struct 2007, 36:131-50.

5. Knipscheer P, Sixma TK: Protein-protein interactions regulate Ubl conjugation. Curr Opin Struct Biol 2007, 17:665-73.

6. Welchman RL, Gordon C, Mayer RJ: Ubiquitin and ubiquitin-like proteins as multifunctional signals. Nat Rev Mol Cell Biol 2005, 6:599-609.

7. Ikeda F, Dikic I: Atypical ubiquitin chains: new molecular signals. 'Protein Modifications: Beyond the Usual Suspects' review series. EMBO Reports 2008, 9:536-42.

8. Huang DT, Hunt HW, Zhuang M, Ohi MD, Holton JM, Schulman BA: Basis for a ubiquitin-like thioester switch toggling EI-E2 affinity. Nature 2007, 445:394-8.

FI000 Factor 9.8 Exceptional

Evaluated by Zhen-Qiang Pan 2 Feb 2007, Jane Endicott 9 Feb 2007, Frank Sicheri 10 Apr 2007

9. Eletr ZM, Huang DT, Duda DM, Schulman BA, Kuhlman B: E2 conjugating enzymes must disengage from their EI enzymes before E3-dependent ubiquitin and ubiquitin-like transfer. Nat Struct Mol Biol 2005, I 2:933-4.
10. Lee I, Schindelin H: Structural insights into El-catalyzed ubiquitin activation and transfer to conjugating enzymes. Cell 2008, 134:268-78.

FI000 Factor 8.0 Exceptional

Evaluated by Zhen-Qiang Pan with Antonio Sarikas I Sep 2008, Hao Wu with Steven M Damo I Oct 2008

II. Walden H, Podgorski MS, Huang DT, Miller DW, Howard RJ, Minor DL Jr., Holton JM, Schulman BA: The structure of the APPBPIUBA3-NEDD8-ATP complex reveals the basis for selective ubiquitin-like protein activation by an El. Mol Cell 2003, 1 2: 1427-37.

12. Lois LM, Lima CD: Structures of the SUMO EI provide mechanistic insights into SUMO activation and E2 recruitment to EI. EMBO J 2005, 24:439-5I.

13. Willems AR, Schwab M, Tyers M: A hitchhiker's guide to the cullin ubiquitin ligases: SCF and its kin. Biochim Biophys Acta 2004, 1695: 133-70.

14. Zheng N, Schulman BA, Song L, Miller JJ, Jeffrey PD, Wang P, Chu C, Koepp DM, Elledge SJ, Pagano M, Conaway RC, Conaway JW, Harper JW, Pavletich NP: Structure of the Cul I-RbxI-Skp I-F boxSkp2 SCF ubiquitin ligase complex. Nature 2002, 416:703-9.

FI000 Factor 8.6 Exceptional

Evaluated by David Lambright 22 Apr 2002, Tom Rapoport 29 Apr 2002, David Barford I5 May 2002, Yigong Shi 27 May 2002, Tony Crowther 28 May 2002, David Morgan 10 Jun 2003

15. Saha A, Deshaies RJ: Multimodal activation of the ubiquitin ligase SCF by Nedd8 conjugation. Mol Cell 2008, 32:2I-3I.

16. Goldenberg SJ, Cascio TC, Shumway SD, Garbutt KC, Liu J, Xiong Y, Zheng N: Structure of the Cand I-CulI-Rocl complex reveals regulatory mechanisms for the assembly of the multisubunit cullin-dependent ubiquitin ligases. Cell 2004, I 19:5 I7-28.

FI000 Factor 6.0 Must Read

Evaluated by Yunde Zhao 16 Nov 2004

17. Duda DM, Borg LA, Scott DC, Hunt HW, Hammel M, Schulman BA: Structural insights into NEDD8 activation of cullin-RING ligases: conformational control of conjugation. Cell 2008, 134:995-1006.

18. Yamoah K, Oashi T, Sarikas A, Gazdoiu S, Osman R, Pan ZQ: Autoinhibitory regulation of SCF-mediated ubiquitination by human cullin I's C-terminal tail. Proc Natl Acad Sci U S A 2008, 105: 12230-5

19. Szczepanowski RH, Filipek R, Bochtler M: Crystal structure of a fragment of mouse ubiquitin-activating enzyme. J Biol Chem 2005, 280:22006-II.

20. Schulman BA, Carrano AC, Jeffrey PD, Bowen Z, Kinnucan ER, Finnin MS, Elledge SJ, Harper JW, Pagano M, Pavletich NP: Insights into SCF ubiquitin ligases from the structure of the SkpI-Skp2 complex. Nature 2000, 408:38I-6.

21. Zheng N, Wang P, Jeffrey PD, Pavletich NP: Structure of a c-CblUbcH7 complex: RING domain function in ubiquitin-protein ligases. Cell 2000, 102:533-9. 